\title{
Tea for Minds and Hearts: A Scoping Review
}

\author{
Dr. Emma Derbyshire ${ }^{1, *}$, Dr. Timothy J Bond², Dr. Gill Jenkins ${ }^{3}$ \\ ${ }^{1}$ Nutritional Insight, Epsom, Surrey, United Kingdom \\ ${ }^{2}$ Tea Advisory Panel; Tea and Herbal Solutions; 103 Warwick Avenue, Bedford, MK40 2DH, UK \\ ${ }^{3}$ General Practitioner (GP), Nuffield Bristol Hospital, 3 Clifton Hill, Bristol BS8 1BN, United Kingdom
}

*Corresponding author: Emma Derbyshire, Nutritional Insight, Epsom, Surrey, United Kingdom, E-mail: emma@nutritional-insight.co.uk

Received: 03 Dec, 2021 | Accepted: 17 Jan, 2022 | Published: 04 Feb, 2022

Citation: Derbyshire E, Bond TJ, Jenkins G (2022) Tea for Minds and Hearts: A Scoping Review. Nutr Food Technol Open Access 8(1): dx.doi.org/10.16966/2470-6086.176

Copyright: (c) 2022 Derbyshire E, et al. This is an open-access article distributed under the terms of the Creative Commons Attribution License, which permits unrestricted use, distribution, and reproduction in any medium, provided the original author and source are credited.

\begin{abstract}
Tea is a ubiquitous and staple beverage for many people globally. It is well documented that mental well being and cardiovascular health are both recognized as being important in determining general health. The two are intricately linked as people with mental health issues are more likely to have heart disease. We systematically searched the PubMed database and selected studies by predefined eligibility criteria. The reviewed studies presented a large body of evidence indicating that black tea drinking could benefit markers of heart health, including vascular and endothelial function. There is emerging evidence that tea drinking (black and green) and aromatic inhalation of certain teas (e.g., Darjeeling and Assam teas) could aid relaxation, lower stress, dementia risk and cognitive decline and potentially improve attention and psychomotor speed in advanced age. Further human trials are warranted.
\end{abstract}

Keywords: Black tea; Cardiovascular disease; Cognition; Emotions; Green tea; Heart health; Mental wellbeing; Memory

Abbreviations: CgA: Chromogranin-A (salivary); CHD: Coronary Heart Disease; Cl: Confidence Interval; COVID 19: Coronavirus Disease 2019; CVD: Cardiovascular Disease; DBP: Diastolic Blood Pressure; EGCG: Epigallocatechin Gallate; FBF: Forearm Blood Flow; FMD: Flow-Mediated Dilation; IHD: Ischaemic Heart Disease; MCE: Major Coronary Events; NF-кB: Nuclear Factor-Kappa B; NO: Nitric Oxide; OR: Odds Ratio; P: Probability; PP: Pulse Pressure; RCTs: Randomised Controlled Trials; SBP: Systolic Blood Pressure; UK: United Kingdom

\section{Introduction}

Globally, an excess of people suffer from mental health issues contributing to one of the largest proportions of the global disease burden [1]. The world health organization now recognizes that there is "no health without mental health", reporting that mental conditions are responsible for $30-40 \%$ of chronic sick leave and around 3\% of Gross Domestic Product with action needed in this important field [2].

In the United Kingdom (UK) it was reported in 2021 that around 1 in 4 people experience mental health issues at some point in the year and 1 in 6 experienced a mental health issue weekly [3]. In total, poor mental health is projected to cost the world economy $\$ 6$ trillion by 2030 due to poor health and reduced productivity [4].

Cardiovascular disease (CVD) is a well-recognised chronic disease with pre-existing CVD now also seemingly being linked to worse outcomes and increased mortality in patients with COVID-19 [5]. Over the past two decades well established risk factors for CVD have been accruing in younger adults-poor diets, obesity and physical inactivity, alongside other temporal risk factors such as electronic cigarettes, and recreational drug use [6]. Ten predominant CVD risk factors are now well recognized which include: unhealthy nutrition, obesity, physical inactivity, and genetics e.g., familial hypercholesterolemia, hyperglycemia, dyslipidemia, high blood pressure, smoking, thrombosis, kidney dysfunction and risk to specific populations (gender, older age, and race/ethnicity) [7].
A fact still unknown to many, CVD remains the leading cause of death amongst women, with premature menopause in particular being linked to an increased risk of coronary heart disease [8]. In the UK alone 7.6 million people live with heart and circulatory conditions and there are over 100,000 hospital admissions annually due to heart attacks and 450 deaths daily due to a heart or circulatory disease [9]. In England alone CVD-related healthcare costs are estimated to be around $£ 7.4$ billion annually, with annual costs to the wider economy being even higher-an estimated $£ 15.8$ billion [10]. It is also thought that acute CVD could be being overlooked because of the COVID-19 pandemic [11].

It is important to consider that the mind and heart are intricately linked. Depression, even at levels lower than those indicative of 'clinical depression' have been associated with increased risk of CVD [12]. Patients with major depressive disorder are known to have a higher prevalence of CVD, with neovascular health (development of new blood vessels especially in tissues where circulation has been impaired by disease or trauma) gaining increasing attention as one possible underlying mechanism [13]. Given the present set of circumstances, this publication aims to provide an updated review on the role of tea in relation to mind and heart health.

\section{Methods}

The National Centre for Biotechnology Information (NCBI) search engine (PubMed.gov) was used to extract relevant publications. 
English-language human studies published between $01^{\text {st }}$ January 2000 and $07^{\text {th }}$ September 2021 were screened. Publications were included if they used tea (Camellia sinensis) and studied specified health outcomes related to the mind and heart. The first search, which used the search term "Tea" combined with "Mind", "Cognition", "Brain health", "Memory" and "Emotions", was first undertaken. The second search applied the term "Tea" combined with "Heart health", "Cardiovascular disease" or "Endothelial function". Reference lists of key papers were also searched.

Both searches (for the mind and heart) were initially restricted to human studies and randomised controlled trials (RCTs). RCTs are regarded as the 'gold standard' for determining efficacy of research, reducing the risk of bias whilst examining cause and effect relationships between interventions and outcomes [14]. Exclusion criteria for both searches included: pilot studies, trials using tea 'capsules' or 'supplements', where outcomes did not relate directly to the mind or heart, or when a full text was unavailable. A separate second search was then undertaken using the same search terms and inclusion/ exclusion criterion for 'observational studies' to encompass evidence from these.

\section{Results}

\section{Randomised controlled trials}

Tea and the mind: At least four RCTs have focused on tea ingestion in relation to outcomes related to the mind (Table 1). Yoto A, et al. and colleagues conducted two studies focusing on tea and salivary chromogranin-A (CgA) levels - a protein marker of stress $[15,16]$. Using a cross-over, single-blind, randomised design, two black tea aromas (Darjeeling and Assam) or hot water as the control were inhaled [16]. Inhaling black tea aromas (both forms) reduced salivary CgA concentration levels 30 minutes after mental stress load tasks [16]. Darjeeling tea aroma appeared to improve mood before mental stress load and reduced the anxiety and/or tension score immediately after the first exposure [16]. Yoto A, et al. (2014) [15] using a similar cross-over, single blind, randomised design, allocated 18 healthy adults to ingest either one $250 \mathrm{ml}$ cup of sagara green tea, shaded white tea [shaded for so long it loses its colour as opposed to commercial 'white tea', which is produced via a process of solar withering followed by indoor withering or water as a control [15]. Shaded white tea ingestion prevented increases in salivary CgA concentration levels after exposure to mental stress load tasks and also lowered total mood disturbance scores [15]. These findings implied that inhaling or ingesting certain tea forms could help to reduce markers of stress which in these instances were CgA $[15,16]$.

Other work conducted by Steptoe and colleagues recruited 75 healthy non-smoking males, allocating these to drink the equivalent to four cups of strong black tea (or a caffeinated placebo) daily over 6-weeks [17]. In these population 6-weeks of black tea consumption resulted in greater subjective relaxation ratings and lower post-task cortisol levels when compared with the placebo [17]. The authors concluded that black tea ingestion could benefit health by facilitating stress recovery [17]. Other work by Hindmarch I, et al. (2000) found that drinking the equivalent to 1 to 2 cups of tea daily (containing 37.5 or $75 \mathrm{mg}$ caffeine) induced similar alerting effects to coffee but was less likely to disrupt sleep [18].

Tea and the heart: A growing body of evidence (at least 13 RCTs) have studied inter-relationships between tea drinking and aspects of heart health (Table 2). The majority of RCTs (at least 11 studies) focused on black tea [19-29]. Two RCTs focused specifically on green tea ingestion $[30,31]$.

Focusing on black tea studies, two focused on vascular function $[19,20]$ and two studied heart rates $[19,23]$. Ahmad and colleagues recruited 17 healthy young adults (mean age 22.4 years) finding that black tea increased flow-mediated dilation compared with the hot water control [19]. The addition of milk, however, appeared to modulate the impact of black tea consumption on vascular function and increased SBP and DBP [19]. Amongst middle-aged adults (mean age 58 years) drinking $200 \mathrm{ml}$ black tea improved cut aneous vascular responses, possibly via the activation of endothelium mediators such as nitric oxide [20]. In relation to heart rate, Ahmad AF, et al. (2018) reported that both black tea and black tea with milk reduced heart rate compared with the hot water control [19]. Hodgson JM, et al. (2013) also observed that heart rate was significantly lower for black tea providing $429 \mathrm{mg}$ polyphenols during early-morning and nighttime periods compared to the control [23].

Table 1: Tea and the Mind-Key RCT Publications.

\begin{tabular}{|c|c|c|c|c|c|}
\hline Reference & $\begin{array}{c}\text { Study } \\
\text { Population }\end{array}$ & Study Design & Tea Intervention & Outcome of Interest & Main Findings \\
\hline $\begin{array}{l}\text { Yoto A, et al. } \\
\text { (2018) [16] Japan }\end{array}$ & $\mathrm{n}=18$ adults. & $\begin{array}{l}\text { Cross-over, single- } \\
\text { blind, randomised } \\
\text { design. }\end{array}$ & $\begin{array}{l}\text { Darjeeling, Assam, or } \\
\text { hot water inhalation. }\end{array}$ & $\begin{array}{c}\text { Salivary } \\
\text { chromogranin-A } \\
\text { levels-a stress } \\
\text { marker }\end{array}$ & $\begin{array}{l}\text { Darjeeling tea aroma appeared to improve } \\
\text { mood before mental stress load. Inhaling } \\
\text { black tea aroma could diminish stress levels } \\
\text { triggered by arithmetic mental stress tasks. }\end{array}$ \\
\hline $\begin{array}{l}\text { Yoto A, et al. } \\
\text { (2014) [15] Japan }\end{array}$ & $\mathrm{n}=18$ students. & $\begin{array}{l}\text { Three experimental } \\
\text { trials on different } \\
\text { days at intervals of } \\
7 \text { days. }\end{array}$ & $\begin{array}{c}1 \text { cup }(250 \mathrm{ml}) \text { of } \\
\text { green tea (Sagara), } \\
\text { shaded white tea or } \\
\text { hot water. }\end{array}$ & $\begin{array}{l}\text { Salivary } \\
\text { chromogranin-A } \\
\text { levels-a stress } \\
\text { marker. }\end{array}$ & $\begin{array}{l}\text { CgA level increased after the mental tasks. } \\
\text { Green tea intake inhibited this increase. } \\
\text { The anti-stress effect was even greater after } \\
\text { shaded white tea consumption. }\end{array}$ \\
\hline $\begin{array}{l}\text { Steptoe A, et al. } \\
\text { (2007) [17] UK }\end{array}$ & $\begin{array}{l}\mathrm{n}=75 \text { healthy } \\
\text { non-smoking } \\
\text { males. }\end{array}$ & $\begin{array}{l}\text { 4-week wash out and } \\
\text { then 6-week trial. }\end{array}$ & $\begin{array}{c}4 \text { cups of strong black } \\
\text { tea (equivalent to) } \\
\text { ingested daily. }\end{array}$ & $\begin{array}{l}\text { Psychophysiological } \\
\text { stress responsivity, } \\
\text { post-stress recovery. }\end{array}$ & $\begin{array}{l}\text { The active tea group had lower post-task } \\
\text { cortisol levels compared with placebo } \\
(P=0.032) \text {, and a relative increase in } \\
\text { subjective relaxation during the post-task } \\
\text { recovery period ( } P=0.036) \text {. }\end{array}$ \\
\hline $\begin{array}{l}\text { Hindmarch I, et } \\
\text { al. (2000) [18] UK }\end{array}$ & $\begin{array}{l}\mathrm{n}=30 \text { healthy } \\
\text { adults. }\end{array}$ & $\begin{array}{l}\text { 5-way randomised } \\
\text { crossover design. }\end{array}$ & $\begin{array}{l}1 \text { or } 2 \text { cups of tea } \\
\text { (containing } 37.5 \mathrm{mg} \\
\text { or } 75 \mathrm{mg} \text { caffeine), } \\
\text { coffee or water. }\end{array}$ & Alertness. & $\begin{array}{c}\text { Day-long tea consumption produced similar } \\
\text { alerting effects to coffee, despite lower } \\
\text { caffeine levels, but was less likely to disrupt } \\
\text { sleep. }\end{array}$ \\
\hline
\end{tabular}

Key: CgA: Chromogranin-A. 
Table 2: Tea and the Heart-Key RCT Publications.

\begin{tabular}{|c|c|c|c|c|c|}
\hline Reference & Study Population & Study Design & Tea Intervention & $\begin{array}{l}\text { Outcome of } \\
\text { Interest }\end{array}$ & Main Findings \\
\hline $\begin{array}{l}\text { Al-Shafei AIM, et } \\
\text { al. (2019) [30], } \\
\text { Egypt }\end{array}$ & $\begin{array}{c}\mathrm{n}=100 \text { adults } 53 \pm 4 \\
\text { years. }\end{array}$ & $\begin{array}{l}\text { 4-month randomised } \\
\text { crossover study. }\end{array}$ & $\begin{array}{c}\text { 4x } 250 \text { ml green tea or } \\
\text { hot water (placebo). } \\
\text { Drank without sugar and } \\
\text { milk. }\end{array}$ & $\begin{array}{l}\text { Pulse pressure, } \\
\text { left ventricular } \\
\text { hypertrophy. }\end{array}$ & $\begin{array}{c}\text { SBP, DBP, and PP were significantly } \\
\text { lowered by } 5.4 \%, 4.1 \% \text {, and } 7.7 \% \text { from the } \\
\text { baseline values after } 4 \text {-weeks of green tea } \\
\text { treatment. }\end{array}$ \\
\hline $\begin{array}{l}\text { Ahmad AF, et } \\
\text { al. (2018) [19], } \\
\text { Australia }\end{array}$ & $\begin{array}{l}n=17 \text { adults, } 22.4 \\
\text { years } \pm 3.0 \text { years. }\end{array}$ & $\begin{array}{l}\text { 4-week controlled } \\
\text { randomised crossover } \\
\text { study. }\end{array}$ & $\begin{array}{l}\text { Hot water, black tea, or } \\
\text { black tea with milk }\end{array}$ & Vascular function. & $\begin{array}{l}\text { Black tea increased FMD compared to the } \\
\text { hot water control }(P<0.0001) \text {. Black tea } \\
(P<0.001) \text { and black tea with milk }(P=0.001) \\
\text { lowered heart rate compared to hot water. }\end{array}$ \\
\hline $\begin{array}{l}\text { Woodward KA, et } \\
\text { al. (2018) [20] UK }\end{array}$ & $\begin{array}{c}n=20 \text { healthy males, } \\
58 \pm 5 \text { years. }\end{array}$ & $\begin{array}{l}\text { 7-days apart in } \\
\text { a randomised, } \\
\text { controlled, double- } \\
\text { blind, cross-over } \\
\text { design. }\end{array}$ & $\begin{array}{l}\text { 1x cup black tea or } \\
\text { placebo }(200 \mathrm{ml})\end{array}$ & Vascular function. & $\begin{array}{l}\text { Acute tea ingestion enhanced cutaneous } \\
\text { vascular responses in healthy, middle-aged } \\
\text { participants, possibly via a mechanism } \\
\text { related to activation of endothelium- } \\
\text { derived chemical mediators, such as NO. }\end{array}$ \\
\hline $\begin{array}{l}\text { Grassi D, et al. } \\
\text { (2016) [29] Italy }\end{array}$ & $\begin{array}{c}\mathrm{n}=19 \text { hypertensive } \\
\text { patients. }\end{array}$ & $\begin{array}{l}\text { 8-day randomized, } \\
\text { double-blind, } \\
\text { controlled, cross-over } \\
\text { study. }\end{array}$ & $\begin{array}{l}2 x \text { cups black tea } \\
\text { beverage daily ( } 150 \\
\text { mg polyphenols) or a } \\
\text { placebo. }\end{array}$ & $\begin{array}{l}\text { Endothelial } \\
\text { progenitor cells, } \\
\text { flow mediated } \\
\text { dilatation. }\end{array}$ & $\begin{array}{c}\text { Tea increased FMD } 1,2,3 \text {, and } 4 \mathrm{~h} \text { after } \\
\text { consumption, with maximal response } 2 \mathrm{~h} \\
\text { after intake }(P<0.0001) .\end{array}$ \\
\hline $\begin{array}{l}\text { Greyling A, et } \\
\text { al. (2016) [21] } \\
\text { Netherlands }\end{array}$ & $\begin{array}{c}n=20 \text { healthy adults, } \\
45-75 \text { years. }\end{array}$ & \begin{tabular}{|} 
2-hr after \\
consumption-double- \\
blind, randomised, \\
placebo-controlled \\
crossover intervention \\
study.
\end{tabular} & $\begin{array}{l}\text { 2-3X cups black tea } \\
\text { providing } 400 \mathrm{mg} \\
\text { flavonoids or colour/ } \\
\text { taste matched placebo. }\end{array}$ & $\begin{array}{l}\text { Resistance artery } \\
\text { endothelial } \\
\text { function. }\end{array}$ & $\begin{array}{c}\text { There was no evidence that acute intake of } \\
\text { black tea significantly altered endothelium- } \\
\text { dependent vasodilation of forearm } \\
\text { resistance arteries. }\end{array}$ \\
\hline $\begin{array}{l}\text { Grassi D, et al. } \\
\text { (2015) [28] Italy }\end{array}$ & $\begin{array}{l}\mathrm{n}=19 \text { hypertensive } \\
\text { patients }\end{array}$ & $\begin{array}{l}\text { 8-day randomized, } \\
\text { double-blind, } \\
\text { controlled, cross-over } \\
\text { study. }\end{array}$ & $\begin{array}{l}2 x \text { cups black tea } \\
\text { beverage daily ( } 150 \\
\text { mg polyphenols) or a } \\
\text { placebo. }\end{array}$ & $\begin{array}{l}\text { Blood pressure, } \\
\text { wave reflections }\end{array}$ & $\begin{array}{l}\text { Black tea significantly decreased SBP } \\
\text { and DBP. Reflection index and stiffness } \\
\text { index decreased after tea consumption } \\
\qquad(\mathrm{P}<0.0001) \text {. }\end{array}$ \\
\hline $\begin{array}{l}\text { Schreuder THA, } \\
\text { et al. (2014) [22] } \\
\text { Netherlands }\end{array}$ & $\begin{array}{l}\mathrm{n}=20 \text { healthy } \\
\text { subjects. }\end{array}$ & $\begin{array}{l}\text { 7-day } x 2 \text { randomised, } \\
\text { crossover study. }\end{array}$ & $\begin{array}{l}3 x \text { cups black tea black } \\
\text { tea or tea abstinence. }\end{array}$ & $\begin{array}{l}\text { Brachial artery } \\
\text { flow-mediated } \\
\text { dilation, ischaemia- } \\
\text { reperfusion. }\end{array}$ & $\begin{array}{l}\text { Tea consumption resulted in an immediate } \\
\text { increase in FMD\% (pre-consumption: } 5.8 \text {; } \\
\text { post-consumption: } 7.2 \mathrm{P}<0.01 \text { ). No change } \\
\text { occurred after hot water ingestion. }\end{array}$ \\
\hline $\begin{array}{l}\text { Hodgson JM, et } \\
\text { al. (2013) [23] } \\
\text { Australia }\end{array}$ & $\mathrm{n}=111$ adults. & $\begin{array}{l}\text { Randomised placebo- } \\
\text { controlled double- } \\
\text { blind parallel designed } \\
\text { trial. }\end{array}$ & $\begin{array}{c}3 x \text { cups black tea over } \\
1 \text { day (powdered black } \\
\text { tea solids containing } 429 \\
\text { mg of polyphenols) or a } \\
\text { control. }\end{array}$ & $\begin{array}{l}\text { Blood pressure, } \\
\text { heart rate. }\end{array}$ & $\begin{array}{l}\text { Heart rate was significantly lower for tea } \\
\text { compared to control during the night-time } \\
\text { and early-morning periods }(-2.0(95 \% \mathrm{Cl} \text {, } \\
-3.2,-0.8) \text { bpm, and }-1.9(95 \% \mathrm{Cl},-3.7,-0.2) \\
\text { bpm, respectively), but not during the } \\
\text { daytime. }\end{array}$ \\
\hline $\begin{array}{l}\text { Grassi D, et al. } \\
\text { (2009) [24] Italy }\end{array}$ & n=19 healthy males. & $\begin{array}{l}5 \times 1 \text { week periods in a } \\
\text { randomised, double- } \\
\text { blind, controlled, } \\
\text { cross-over design. }\end{array}$ & $\begin{array}{l}2 x \text { black tea intake }- \text { five } \\
\text { treatments with a twice } \\
\text { daily intake of black tea } \\
(0,100,200,400 \text { and } 800 \\
\text { mg tea flavonoids/day). }\end{array}$ & $\begin{array}{l}\text { Flow-mediated } \\
\text { dilation. }\end{array}$ & $\begin{array}{l}100 \mathrm{mg} / \text { day (less than } 1 \text { cup of tea) } \\
\text { increased FMD compared with control } \\
\qquad(P=0.0113) .\end{array}$ \\
\hline $\begin{array}{l}\text { Nagaya N, et al. } \\
\text { (2004) [31] Japan }\end{array}$ & $\begin{array}{l}\mathrm{n}=20 \text { healthy } \\
\text { smokers. }\end{array}$ & $\begin{array}{c}2 \text { days, } 1 \text { week apart - } \\
\text { randomised crossover } \\
\text { study. }\end{array}$ & $\begin{array}{c}400 \mathrm{ml} \text { green tea or hot } \\
\text { water. }\end{array}$ & $\begin{array}{l}\text { Endothelial } \\
\text { dysfunction. }\end{array}$ & $\begin{array}{l}\text { Green tea consumption significantly } \\
\text { increased FBF during reactive hyperaemia } \\
\qquad(P<0.001)\end{array}$ \\
\hline $\begin{array}{l}\text { Hodgson JM, et al. } \\
(2002)[25]\end{array}$ & $\begin{array}{l}\mathrm{n}=21 \text { subjects with } \\
\text { mildly elevated } \\
\text { serum cholesterol } \\
\text { or triacylglycerol. }\end{array}$ & 4-week trial. & $\begin{array}{l}5 x \text { cups black tea or hot } \\
\text { water daily. }\end{array}$ & $\begin{array}{l}\text { Brachial artery } \\
\text { vasodilator } \\
\text { function. }\end{array}$ & $\begin{array}{l}\text { Regular ingestion of black tea resulted } \\
\text { in a significant increase in endothelium- } \\
\text { dependent dilatation ( } 2.3 \% ; \mathrm{P}=0.008) \text { and } \\
\text { in a significant increase in endothelium- } \\
\text { independent dilatation ( } 4.2 \% ; \mathrm{P}=0.03) \text {, } \\
\text { compared with ingestion of hot water. }\end{array}$ \\
\hline $\begin{array}{l}\text { Duffy SJ, et al. } \\
\text { (2001) [26] USA }\end{array}$ & $\begin{array}{c}\mathrm{n}=66 \text { patients with } \\
\text { coronary artery } \\
\text { disease. }\end{array}$ & $\begin{array}{l}\text { 4-week randomised- } \\
\text { controlled crossover } \\
\text { study. }\end{array}$ & $\begin{array}{c}900 \mathrm{ml} \text { black tea or hot } \\
\text { water daily. }\end{array}$ & $\begin{array}{l}\text { Endothelial } \\
\text { dysfunction. }\end{array}$ & $\begin{array}{c}\text { Short-and long-term tea consumption } \\
\text { increased endothelium- dependent flow- } \\
\text { mediated dilation of the brachial artery, } \\
\text { whereas consumption of water had no } \\
\text { effect }(P<0.001) \text {. }\end{array}$ \\
\hline $\begin{array}{l}\text { Hodgson JM, et } \\
\text { al. (2001) [27] } \\
\text { Australia }\end{array}$ & $\mathrm{n}=22$ adults. & $\begin{array}{l}\text { 4-week randomised- } \\
\text { controlled crossover } \\
\text { study. }\end{array}$ & $\begin{array}{l}5 x \text { cups black tea or hot } \\
\text { water daily. }\end{array}$ & $\begin{array}{l}\text { Haemostasis, } \\
\text { cell adhesion } \\
\text { molecules. }\end{array}$ & $\begin{array}{l}\text { Black tea resulted in lower soluble } P \text {-select } \\
\text { in }(P=0.01) \text { versus hot water but did not } \\
\text { influence other adhesion molecules. }\end{array}$ \\
\hline
\end{tabular}

Key: BP: Blood Pressure; Cl: Confidence Interval; DBP: Diastolic Blood Pressure; FBF: Forearm Blood Flow; FMD: Flow-Mediated Dilation; NO: Nitric Oxide; P: Probability; PP: Pulse Pressure; SBP: Systolic Blood Pressure. 
Table 3: Tea, the Mind and Heart-Key Observational Publications.

\begin{tabular}{|c|c|c|c|c|}
\hline Reference & Study Population & Study Design & $\begin{array}{l}\text { Outcome of } \\
\text { Interest }\end{array}$ & Main Findings \\
\hline \multicolumn{5}{|c|}{ The Mind } \\
\hline $\begin{array}{l}\text { Yao Y, et al. (2021) } \\
\text { [39] China }\end{array}$ & $n=13,115$ adults. & $\begin{array}{l}\text { Chinese Longitudinal } \\
\text { Healthy Longevity Survey }\end{array}$ & Depression. & $\begin{array}{l}\text { Compared with the group of no tea drinkers, } \\
\text { ORs of depressive symptoms for daily green } \\
\text { tea, fermented tea, and floral tea intake were } \\
0.85(95 \% \mathrm{Cl}: 0.76-0.95), 0.87(95 \% \mathrm{Cl}: 0.76- \\
0.99) \text {, and } 0.70(95 \% \mathrm{Cl}: 0.59-0.82) \text { amongst } \\
\text { older Chinese adults. }\end{array}$ \\
\hline $\begin{array}{l}\text { Okello EJ, et al. } \\
\text { (2020) [40] UK }\end{array}$ & $n=676,85+$ years. & $\begin{array}{l}\text { Community based } \\
\text { longitudinal study. }\end{array}$ & $\begin{array}{l}\text { Attention, } \\
\text { psychomotor } \\
\text { speed. }\end{array}$ & $\begin{array}{l}\text { High level of tea consumption was associated } \\
\text { with significantly better attention (focused and } \\
\text { sustained attention), and psychomotor speed } \\
\text { (complex tasks only) over five-year follow-up. }\end{array}$ \\
\hline $\begin{array}{l}\text { Shirai Y, et al. (2020) } \\
\text { [41] Japan }\end{array}$ & $\begin{array}{c}n=620 \text { males, } n=685 \text { females, } \\
60-85 \text { years. }\end{array}$ & Prospective cohort study. & Cognitive decline. & $\begin{array}{l}\text { Green tea lowered the risk of cognitive decline. } \\
\text { Compared with participants who consumed } \\
\text { green tea <once/d, the multivariable hazard } \\
\text { ratio }(95 \% \mathrm{Cl} \text { ) was } 0 \cdot 70,0 \cdot 71 \text { and } 0 \cdot 72 \text { among } \\
\text { those who ingested green tea once/d, } 2-3 \\
\text { times/d and } \geq 4 \text { times/d, respectively. }\end{array}$ \\
\hline $\begin{array}{l}\text { Lee CY, et al. (2017) } \\
\text { [32] Taiwan }\end{array}$ & $\begin{array}{l}\mathrm{n}=10432 \text { residents, } \\
\mathrm{n}=2049 \text { with mild cognitive } \\
\text { impairment, } \mathrm{n}=929 \text { with } \\
\text { dementia, } \mathrm{n}=7035 \text { without } \\
\text { dementia or mild cognitive } \\
\text { impairment. }\end{array}$ & $\begin{array}{c}\text { Cross-sectional } \\
\text { observational study. }\end{array}$ & Dementia. & $\begin{array}{l}\text { Inverse associations between green tea and } \\
\text { dementia (OR } 0.51,95 \% \mathrm{Cl} 0.34-0.75 \text { ) and } \\
\text { other types of tea consumption (OR 0.41, 95\% } \\
\qquad \mathrm{Cl} 0.28-0.60 \text { ) were found. }\end{array}$ \\
\hline $\begin{array}{l}\text { Bryan J, et al. } \\
\text { (2012) [33] Australia }\end{array}$ & $\begin{array}{l}\mathrm{n}=95 \text { professional and } \\
\text { academic staff. }\end{array}$ & $\begin{array}{c}\text { Cross-sectional } \\
\text { observational study. }\end{array}$ & Mood. & $\begin{array}{l}\text { Ingestion of non-caffeinated beverages was } \\
\text { associated with increased relaxation and } \\
\text { recovery from work. Tea and other caffeinated } \\
\text { beverages, however, were found to enhance } \\
\text { the negative effects of evening recovery and } \\
\text { morning mood on mindfulness during the day. }\end{array}$ \\
\hline $\begin{array}{l}\text { Shimbo M, et al. } \\
\text { (2005) [34] Japan }\end{array}$ & $n=600$ aged $20-69$ years. & $\begin{array}{c}\text { Cross-sectional } \\
\text { observational study. }\end{array}$ & Mental health. & $\begin{array}{l}\text { The ingestion of brewed green tea was not } \\
\text { statistically associated with any decrease in } \\
\text { risk of mental ill-health among either males or } \\
\text { females. }\end{array}$ \\
\hline \multicolumn{5}{|c|}{ The Heart } \\
\hline $\begin{array}{l}\text { Chen Y, et al. (2019) } \\
\text { [35] China }\end{array}$ & $\begin{array}{l}\mathrm{n}=267 \text { cases of } \mathrm{CHD} \text { and } \\
235 \text { non-CHD controls were } \\
\text { enrolled. }\end{array}$ & $\begin{array}{l}\text { Hospital-based case- } \\
\text { control study. }\end{array}$ & $\begin{array}{l}\text { Coronary Heart } \\
\text { Disease. }\end{array}$ & $\begin{array}{c}\text { CHD risk was reduced in women who ingested } \\
1-2 \text { cups of tea per day }(\mathrm{OR}=0.291,95 \% \\
\mathrm{Cl}: 0.131-0.643, \mathrm{P}=0.0023) \text {. A tea-drinking } \\
\text { frequency of }>6 \text { days/week was beneficial for } \\
\mathrm{CHD} \text { prevention }(\mathrm{OR}=0.183,95 \% \mathrm{Cl}: 0.049- \\
0.679, \mathrm{P}=0.0112) .\end{array}$ \\
\hline $\begin{array}{l}\text { Li X, et al. (2017) } \\
\text { [36] China }\end{array}$ & $\begin{array}{c}\mathrm{n}=199293 \text { men and } 288082 \\
\text { women aged 30-79 years at } \\
\text { baseline. }\end{array}$ & $\begin{array}{l}\text { Prospective study using } \\
\text { the China Kadoorie } \\
\text { Biobank. }\end{array}$ & $\begin{array}{l}\text { Ischaemic heart } \\
\text { disease. }\end{array}$ & $\begin{array}{l}\text { Daily tea consumption was associated with a } \\
\text { reduced risk of IHD compared with participants } \\
\text { who never consumed tea during the past } 12 \\
\text { months. }\end{array}$ \\
\hline $\begin{array}{l}\text { Liu DC, et al. (2016) } \\
\text { [37] China }\end{array}$ & $\begin{array}{c}n=80, n=401 \text { atrial fibrillation } \\
\text { patients and } 400 \text { controls. }\end{array}$ & Case-control study. & Atrial Fibrillation. & $\begin{array}{l}\text { Green tea intake seemed to be a protective } \\
\text { factor against the incidence of atrial fibrillation } \\
\text { (OR: } 0.349,95 \% \mathrm{Cl}: 0.253-0.483, \mathrm{P}<0.001 \text { ). }\end{array}$ \\
\hline $\begin{array}{l}\text { Chrysohoou C, et al. } \\
\text { (2016) [38] Greece }\end{array}$ & $\begin{array}{c}\mathrm{n}=30 \text { men and } 343 \text { women, } \\
\text { aged } 65 \text { to } 100 \text { years. }\end{array}$ & $\begin{array}{c}\text { Cross-sectional } \\
\text { observational study. }\end{array}$ & $\begin{array}{l}\text { Cardiovascular } \\
\text { Disease. }\end{array}$ & $\begin{array}{c}\text { Tea drinking was inversely associated with CVD } \\
\text { incidence. }\end{array}$ \\
\hline
\end{tabular}

Key: CHD: Coronary Heart Disease; Cl: Confidence Interval; IHD: Ischaemic Heart Disease; MCE: Major Coronary Events; OR: Odds Ratio. 
Other RCTs investigated markers of endothelial function or flowmediated dilation (FMD) [21,22,24-26]. Longer trials tended to report beneficial effects. For example, Grassi D, et al. (2009) conducted a 5 -week trial on 19 healthy males, finding that less than one cup of black tea daily increased flow-mediated dilation (FMD) compared with the control [24]. A 4-week trial recruiting 21 adults with mildly elevated serum cholesterol/triacylglycerol at baseline, found that drinking 5 cups of black tea daily consistently and significantly improved endothelium dilatation indicating that black tea improved the vasodilator function which could be a plausible mechanism for lowering CVD risk [25]. Similarly, in another 4-week trial drinking $900 \mathrm{ml}$ of black tea daily was linked to reversed endothelial vasomotor function in coronary artery disease patients [26]. In a shorter 8-day study Grassi D, et al. and colleagues $(2015 ; 2016)$ found that black tea sachet ingestion (diluted with 100-200 ml hot water) improved levels of functionally active circulating angiogenic cells and FMD, particularly 2-hours after intake [29]. Systolic and diastolic blood pressure, blood pressure and wave reflections also improved [28]. Schreuder THA, et al. (2014) posits that drinking 3 cups of black tea daily significantly improved FMD [22].

Conversely, in a shorter-term trial monitoring vasodilation 2 hours after tea consumption (equivalent to 2-3 cups) there were no effects observed [21]. The authors concluded that longer periods of tea ingestion may be required to observe any effects [21]. One trial sought to evaluate the effect of black tea ingestion (5 cups daily) on cell adhesion molecules, concluding that black tea lowered P-select in which could be another potential underpinning mechanism behind the cardiovascular benefits of tea ingestion [27].

Regarding green tea, Al-Shafei AIM, et al. (2019) recruited 100 adults allocating these to ingest four $250 \mathrm{ml}$ cups of green tea per day (without milk or sugar) or hot water daily over 8-months [30]. At this level of intake, regular green tea consumption induced cardio protective effects - pulse pressure, systolic and diastolic blood pressure reduced significantly by $9.1 \%, 6.6 \%$ and $5.1 \%$, respectively, midway through the study at 4-months [30]. A shorter and smaller trial conducted on two separate days provided healthy male smokers with $400 \mathrm{ml}$ green tea or hot water [31]. Blood pressure and heart rate were unaffected but markers of endothelial dysfunction were reversed green tea consumption significantly improved forearm blood flow during a period of reactive hyperaemia (increased blood flow due to ischemia or arterial blockage) [31].

\section{Observational Evidence}

Several observational studies appear to have focused on outcomes related to the mind [32-34] or heart [35-38]. Longitudinal research found that regular tea drinking (green, fermented, floral) amongst older Chinese adults was associated with fewer depressive symptoms [39]. Another large nationwide survey $(\mathrm{n}=10432)$ conducted across all 19 counties in Taiwan observed that green tea (OR 0.51, 95\% CI 0.340.75 ) and other types of tea (OR $0.41,95 \%$ CI $0.28-0.60$ ) were inversely associated with dementia [32]. Similarly, in the United Kingdom, a longitudinal community study found that higher tea intakes were associated with significantly improved attention and psychomotor speed amongst those aged 85 years or older [40]. Other prospective cohorts [41] found green tea (up to 4 cups daily) to be associated with a reduced risk of cognitive decline in older populations aged 60 to 85 years.

Earlier research using a naturalistic, cross-sectional study design recruited professional and academic staff who logged their beverage intake and registered their work performance [33]. Multilevel modeling analysis found that non-caffeinated drinks, particularly when served without milk or sugar, reduced tiredness and improved perceived work performance, but when caffeinated the beverage appeared to enhance negative effects of evening recovery or mindfulness [33]. Other observational evidence also suggests that caffeinated beverages could potentially impact on mental well being Shimbo M, et al. (2005) conducted a cross-sectional study consisting of 600 adults [34]. After several data adjustments for potential confounders, daily caffeine intake $(100 \mathrm{mg})$ which included black tea, green tea, coffee, and other caffeine-containing beverages was associated with a higher odds of mental health problems amongst females [34].

As with the mind, several observational studies have studied interrelationships between tea drinking and markers of heart health. In Guangzhou, China, tea ingestion (1-2 cups daily or black, oolong or green tea) was associated with reduced coronary heart disease risk amongst females, with a tea-drinking frequency of $>6$ days per week being regarded as beneficial for the prevention of coronary heart disease [35]. Other prospective research using data from the Chinese Kadoorie Biobank found that daily tea consumption (black, oolong or green tea) reduced is chaemic heart disease risk, particularly in rural, healthy weight, non-diabetic adults [36]. Liu DC, et al. (2016) again conducted research in a Chinese population and found that low-dose green tea ingestion, which accounted for the frequency, concentration, and duration of green tea intake, lowered a trial fibrillation incidence [37]. In the Greek Ikaria Island Study (a region with one of the highest global life expectancies), tea drinking was associated with a reduced risk of CVD [38].

\section{Potential mechanisms}

The mind: There have been several theories proposed about how tea could affect the human mind. In particular, the tea components L-theanine and caffeine are understood to have synergistic effects, helping to reduce mind deviation to distractors, such as mind wandering, thus improving attention [42]. Multiple compounds in tea have been found to be effective modulators of dopaminergic activity and the gut-brain axis [43]. Tea constituents present in major tea forms, including L-theanine, polyphenols and polyphenol metabolites, for example, are capable of functioning via multiple pathways collectively helping to reduce depression risk [43].

Systematic analyses have previously concluded that green tea appears to reduce anxiety, improve attention and memory and aspects of brain function e.g., activation of working memory but such effects cannot be pinpointed to a 'single' tea constituent [44]. For green tea, its constituent catechins, including epigallocatechin gallate (EGCG), are proposed to be involved in mechanisms including preventing inflammation, oxidative stress and the abnormal accumulation of fibrous proteins (particularly $A \beta$ and $\alpha$-synuclein) which could contribute to neurodegenerative conditions such as cognitive dysfunction and memory loss [45]. Pioneering research using electroencephalogram technology measuring brain activity showed that 'theta waves' increased significantly 30 minutes to 1 hour after green tea consumption. Scientists proposed that this could be one way in which green tea exerts its potential effects on alertness, attention, and cognitive function [46]. More recently, green tea has been proposed to suppress brain ageing [47]. EGCG and the anine -the bioactive components unique to green tea - are thought to play a role in this by activating nerve cells and reducing stress [47].

The heart: A growing number of potential mechanisms protecting cardiomyocyte function including antioxidant, anti-inflammatory, anti-proliferative effects, reducing blood lipids, alleviating ischemia, 
and favorable modifications to endothelial function have been linked to tea and its bioactive compounds and are reviewed elsewhere $[48,49]$.

Tea polyphenols, (Table 3) in particular, are thought to be responsible for exerting some of these effects [50]. For example, it has been proposed that tea polyphenols can suppress certain inflammatory factors, including nuclear factor-kappa B (NF- $\bigotimes \mathrm{B}$ ) [50]. Catechins, the predominant polyphenols in green tea have been linked to vascular effects via its ability to scavenge free radicals, inhibit enzymes involved in lipid biosynthesis, regulation of vascular tone by activating endothelial nitric oxide and prevention of vascular inflammation [51].

In laboratory models, black tea has been found to attenuate markers of vascular dysfunction, potentially by down-regulating homocysteine enzymes [52]. This is thought to be beneficial as elevated homocysteine levels are typically a marker of CVD risk and can induce endoplasmic reticulum stress in endothelial cells, exacerbating vascular dysfunction [52].

\section{Discussion}

An established body of RCTs and growing body of evidence has focused on tea consumption in relation to the heart and mind, respectively. For the heart, the majority of RCTs (at least 11 trials) have focused on black tea ingestion, with study intakes ranging from 1 to 5 cups daily $[20,25,27]$. An array of outcomes have been investigated, ranging from vascular function, endothelial dysfunction, blood pressure, heart rate, FMD to levels of cell adhesion molecules. Most studies indicate that regular black tea ingestion could attenuate certain underpinning CVD risk factors including blood pressure, vascular and endothelial dysfunction [20,22,24-26,28,29]. Observational research, predominantly conducted in China is suggestive of positive links between tea drinking and aspects of heart health, particularly amongst females $[35,36,38]$. Elsewhere, meta-analytical evidence shows those longer durations of tea intake i.e., $\geq 3$ months, resulted in greater reductions in systolic and diastolic blood pressure [53]. These hypotensive effects were particularly pronounced for green tea compared with black tea in those with higher baseline blood pressure or hypertension [53]. Other meta-analytical work demonstrates that black or green tea ingestion could benefit adults with prehypertension or hypertension, thus potentially aiding secondary prevention of cardiovascular disease [54].

Regarding the mind, the number of RCTs is growing. Strong black tea consumption has been found to improve subjective relaxation ratings and lower post-task cortisol levels when compared with the placebo [17]. Other work showed that drinking the equivalent to 1 to 2 cups of tea daily (containing 37.5 or $75 \mathrm{mg}$ caffeine) induced similar alerting effects to coffee but was less likely to disrupt sleep. In addition, observational evidence implies that tea drinking in general could improve attention and psychomotor speed [40], reduce depression [39] whilst green tea has been associated with reduced cognitive decline [41] and dementia [32] in advanced age.

Some observational studies also suggest that non-caffeinated tea drinking, without milk and sugar could aid relaxation and recovery from work [33]. The role of caffeine derived from beverages, however, remains emergent in relation to mental wellbeing and more research is needed. Higher levels of caffeine consumption have been associated with mental distress in populations of younger populations, especially males $[55,56]$. Ongoing research is needed but provisional findings appear to indicate that herbal tea infusions could be more suitable for aiding relaxation and reducing stress via their inhalation and ingestion $[15-17,33,57]$. On elimitation with some observational studies is that different tea forms are often collated together making clear conclusions a challenge. Future studies need to 'separate out' baseline populations, tea forms and better align study outcomes.

From a social stance, research from surveys has also shown that tea drinking could have extended social and wellbeing implications, with some of these outcomes not yet being studied formally in studies. For example, an Omnibus survey of 1000 UK adults reviewed by the tea advisory panel found that 57 percent of British tea drinkers said that the tea round at work helped them to feel connected to colleagues [58]. Forty-four percent reported that drinking black tea helped them to feel calm and 36 percent said it helped them to tackle a busy day. One-fifth (22\%) of respondents working from home say that they miss the tea break at work and one-third (32\%) admitted that there's a lot of truth to the saying "a cup of tea is the answer to every problem". These qualitative findings suggest that tea drinking has wider social ramifications that could impact on mindset. This would be worthy of future investigation.

\section{Conclusions}

A reasonable conclusion from the available evidence is that including daily tea intake as part of a healthy habitual dietary pattern could be associated with lower cardiovascular disease risks [59]. The UK Eat well Guides presently advises us to drink 6-8 glasses of fluid every day and water, lower fat milk and sugar-free drinks including tea and coffee all count [60]. In essence, data is nebulous at present to specifically conclude whether tea consumption could improve mental well being. Provisional evidence implies that drinking (e.g. black, green, herbal infusions) and inhaling the aroma of certain teas (Darjeeling tea) could aid relaxation, lower markers of stress, dementia risk and cognitive decline and potentially improve attention and psychomotor speed in advanced age. Further trials are now warranted.

\section{Funding sources/sponsors}

The authors received funding provided by the Tea Advisory Panel which is supported by an unrestricted educational grant from the United Kingdom Tea \& Infusions Association (UKTIA), the trade association for the United Kingdom tea and infusions industry. The UKTIA plays no role in producing the outputs of the panel. Independent panel members include nutritionists, biochemists, dietitians, dentist, and doctors.

\section{Conflicts of Interest}

The authors declare no conflicts of interest. The views expressed are those of the authors alone and personnel from the United Kingdom Tea \& Infusions Association (UKTIA) had no role in writing this review.

\section{References}

1. Wainberg ML, Scorza P, Shultz JM, Helpman L, Mootz JJ, et al. (2017) Challenges and Opportunities in Global Mental Health: a Researchto-Practice Perspective. Curr Psychiatry Rep 19: 28.

2. WHO. Mental Health 2021.

3. MHM (2021) Mental Health Midlands. 1 In 4 People In The UK Suffer From Mental Health Issues.

4. The Lancet Global (2020) Mental health matters. Lancet Glob Health: e1352.

5. Nishiga M, Wang DW, Han Y, Lewis DB, Wu JC (2020) COVID-19 and cardiovascular disease: from basic mechanisms to clinical perspectives. Nat Rev Cardiol 17: 543-558.

6. Andersson C, Vasan RS (2018) Epidemiology of cardiovascular disease in young individuals. Nat Rev Cardiol 15: 230-240. 
7. Bays HE, Taub PR, Epstein E, Michos ED, Ferraro RA, et al. (2021) Ten things to know about ten cardiovascular disease risk factors. Am J Prev Cardiol 5: 100149.

8. DiNardo D, McNeil M (2020) Update on Women's Heart Disease. J Womens Health (Larchmt) 29: 1028-1031.

9. BHF (2021) Heart Statistics.

10. Waterall J (2019) Health Matters: Preventing cardiovascular disease. UK Health Security Agency.

11. Huet F, Prieur C, Schurtz G, Gerbaud E, Silberman SM, et al. (2020) One train may hide another: Acute cardiovascular diseases could be neglected because of the COVID-19 pandemic. Arch Cardiovasc Dis 113: 303-307.

12. Harshfield EL, Pennells L, Schwartz JE, Willeit P, Kaptoge S, et al. (2020) Association Between Depressive Symptoms and Incident Cardiovascular Diseases. JAMA 324: 2396-2405.

13. Dudek KA, Albert LD, Kaufmann FN, Tuck E, Lebel M, et al. (2021) Neurobiology of resilience in depression: immune and vascular insights from human and animal studies. Eur J Neurosci 53: 183-221.

14. Hariton E, Locascio JJ (2018) Randomised controlled trials - the gold standard for effectiveness research: Study design: randomised controlled trials. BJOG 125: 1716.

15. Yoto A, Murao S, Nakamura Y, Yokogoshi H (2014) Intake of green tea inhibited increase of salivary chromogranin A after mental task stress loads. J Physiol Anthropol 33: 20.

16. Yoto A, Fukui N, Kaneda C, Torita S, Goto K, et al. (2018) Black tea aroma inhibited increase of salivary chromogranin-A after arithmetic tasks. J Physiol Anthropol 37: 3.

17. Steptoe A, Gibson EL, Vuononvirta R, Williams ED, Hamer M, et al. (2007) The effects of tea on psychophysiological stress responsivity and post-stress recovery: a randomised double-blind trial. Psychopharmacology (Berl) 190: 81-89.

18. Hindmarch I, Rigney U, Stanley N, Quinlan P, Rycroft J, et al. (2000) A naturalistic investigation of the effects of day-long consumption of tea, coffee and water on alertness, sleep onset and sleep quality. Psychopharmacology (Berl) 149: 203-216.

19. Ahmad AF, Rich L, Koch H, Croft KD, Ferruzzi MG, et al. (2018) Effect of adding milk to black tea on vascular function in healthy men and women: a randomised controlled crossover trial. Food Funct 9: 6307-6314.

20. Woodward KA, Hopkins ND, Draijer R, Graaf YD, Low DA, et al. (2018) Acute black tea consumption improves cutaneous vascular function in healthy middle-aged humans. Clin Nutr 37: 242-249.

21. Greyling A, Wolters TLC, Bresser DMD, Roerink SHPP, Riksen NP, et al. (2018) The acute effect of black tea consumption on resistance artery endothelial function in healthy subjects. A randomized controlled trial. Clin Nutr ESPEN 23: 41-47.

22. Schreuder THA, Eijsvogels TMH, Greyling A, Draijer R, Hopman MTE, et al. (2014) Effect of black tea consumption on brachial artery flow-mediated dilation and ischaemia-reperfusion in humans. Appl Physiol Nutr Metab 39: 145-151.

23. Hodgson JM, Woodman RJ, Puddey IB, Mulder T, Fuchs D, et al. (2013) Short-term effects of polyphenol-rich black tea on blood pressure in men and women. Food Funct 4: 111-115.

24. Grassi D, Mulder TP, Draijer R, Desideri G, Molhuizen HO, et al. (2009) Black tea consumption dose-dependently improves flowmediated dilation in healthy males. J Hypertens 27: 774-781.
25. Hodgson JM, Puddey IB, Burke V, Watts GF, Beilin LJ (2002) Regular ingestion of black tea improves brachial artery vasodilator function. Clin Sci (Lond) 102: 195-201.

26. Duffy SJ, Keaney JF, Holbrook M, Gokce N, Swerdloff PL, et al. (2001) Short- and long-term black tea consumption reverses endothelial dysfunction in patients with coronary artery disease. Circulation 104: 151-156.

27. Hodgson JM, Puddey IB, Mori TA, Burke V, Baker RI, et al. (2001) Effects of regular ingestion of black tea on haemostasis and cell adhesion molecules in humans. Eur J Clin Nutr 55: 881-886.

28. Grassi D, Draijer R, Desideri G, Mulder T, Ferri C (2015) Black tea lowers blood pressure and wave reflections in fasted and postprandial conditions in hypertensive patients: a randomised study. Nutrients 7: 1037-1051.

29. Grassi D, Draijer R, Schalkwijk C, Desideri G, Angeli AD, et al. (2016) Black Tea Increases Circulating Endothelial Progenitor Cells and Improves Flow Mediated Dilatation Counteracting Deleterious Effects from a Fat Load in Hypertensive Patients: A Randomized Controlled Study. Nutrients 8: 727

30. Al-Shafei AIM, El-Gendy OAA (2019) Regular consumption of green tea improves pulse pressure and induces regression of left ventricular hypertrophy in hypertensive patients. Physiol Rep 7: e14030.

31. Nagaya $\mathrm{N}$, Yamamoto $\mathrm{H}$, Uematsu $\mathrm{M}$, Itoh $\mathrm{T}$, Nakagawa $\mathrm{K}$, et al. (2004) Green tea reverses endothelial dysfunction in healthy smokers. Heart 90: 1485-1486.

32. Lee CY, Sun Y, Lee HJ, Chen TF, Wang PN ， et al. (2017) Modest Overweight and Healthy Dietary Habits Reduce Risk of Dementia: A Nationwide Survey in Taiwan. J Prev Alzheimers Dis 4: $37-43$

33. Bryan J, Tuckey M, Einöther SJL, Garczarek U, Garrick A, et al. (2012) Relationships between tea and other beverage consumption to work performance and mood. Appetite 58: 339-346.

34. Shimbo M, Nakamura K, Shi HJ, Kizuki M, Seino K, et al. (2005) Green tea consumption in everyday life and mental health. Public Health Nutr 8: 1300-1306.

35. Chen $Y$, Ye Y, Zhang Z, Zhang C, Chen M, et al. (2019) Tea consumption is associated with a reduced risk of coronary heart disease in female but not male populations in Guangzhou, China. Nutr Res Pract 13: 393-398.

36. Li X, Yu C, Guo Y, Bian Z, Si J, et al. (2017) Tea consumption and risk of ischaemic heart disease. Heart 103: 783-789.

37. Liu DC, Yan JJ, Wang YN, Wang ZM, Xie ZY, et al. (2016) Low-dose green tea intake reduces incidence of atrial fibrillation in a Chinese population. Oncotarget 7: 85592-85602.

38. Chrysohoou C, Pitsavos C, Lazaros G, Skoumas J, Tousoulis D, et al. (2016) Determinants of All-Cause Mortality and Incidence of Cardiovascular Disease (2009 to 2013) in Older Adults: The Ikaria Study of the Blue Zones. Angiology 67: 541-548.

39. Yao $Y$, Chen H, Chen L, Ju SY, Yang H, et al. (2021) Type of tea consumption and depressive symptoms in Chinese older adults. BMC Geriatr 21: 331

40. Okello EJ, Mendonça N, Stephan B, Terrera GM, Wesnes K, et al. (2020) Tea consumption and measures of attention and psychomotor speed in the very old: the Newcastle $85+$ longitudinal study. BMC Nutr 6: 57. 
41. Shirai Y, Kuriki K, Otsuka R, Kato Y, Nishita Y, et al. (2020) Green tea and coffee intake and risk of cognitive decline in older adults: the National Institute for Longevity Sciences, Longitudinal Study of Aging. Public Health Nutr 23: 1049-1057.

42. Kahathuduwa CN, Dhanasekara CS, Chin SH, Davis T, Weerasinghe VS, et al. (2018) I-Theanine and caffeine improve target-specific attention to visual stimuli by decreasing mind wandering: a human functional magnetic resonance imaging study. Nutr Res 49: 67-78.

43. Rothenberg DO, Zhang L (2019) Mechanisms Underlying the AntiDepressive Effects of Regular Tea Consumption. Nutrients 11: 1361.

44. Mancini E, Beglinger C, Drewe J, Zanchi D, Lang UE, et al. (2017) Green tea effects on cognition, mood and human brain function: $A$ systematic review. Phytomedicine 34: 26-37.

45. Pervin M, Unno K, Ohishi T, Tanabe H, Miyoshi N, et al. (2018) Beneficial Effects of Green Tea Catechins on Neurodegenerative Diseases. Molecules 23: 1297.

46. Okello EJ, Abadi AM, Abadi SA (2016) Effects of green and black tea consumption on brain wave activities in healthy volunteers as measured by a simplified Electroencephalogram (EEG): A feasibility study. Nutr Neurosci 19: 196-205.

47. Unno K, Nakamura Y (2021) Green Tea Suppresses Brain Aging. Molecules 26: 4897.

48. Deka A, Vita JA (2011) Tea and cardiovascular disease. Pharmacol Res 64: 136-145.

49. Cao SY, Zhao CN, Gan RY, Xu XY, Wei XL, et al. (2019) Effects and Mechanisms of Tea and Its Bioactive Compounds for the Prevention and Treatment of Cardiovascular Diseases: An Updated Review. Antioxidants (Basel) 8: 166.

50. Suzuki Jl, Isobe M, Morishita R, Nagai R (2009) Tea polyphenols regulate key mediators on inflammatory cardiovascular diseases. Mediators Inflamm 2009: 494928.

51. Babu PVA, Liu D (2008) Green tea catechins and cardiovascular health: an update. Curr Med Chem 15: 1840-1850.
52. Cheang WS, Ngai CY, Tam YY, Tian XY, Wong WT, et al. (2015) Black tea protects against hypertension-associated endothelial dysfunction through alleviation of endoplasmic reticulum stress. Sci Rep 5: 10340

53. Roshan MM, Salari A, Ghorbani Z, Ashouri A (2020) The effects of regular consumption of green or black tea beverage on blood pressure in those with elevated blood pressure or hypertension: A systematic review and meta-analysis. Complement Ther Med 51: 102430.

54. Yarmolinsky J, Gon G, Edwards P (2015) Effect of tea on blood pressure for secondary prevention of cardiovascular disease: a systematic review and meta-analysis of randomized controlled trials. Nutr Rev 73: 236-246.

55. Begdache L, Sadeghzadeh S, Derose G, Abrams C (2020) Diet, Exercise, Lifestyle, and Mental Distress among Young and Mature Men and Women: A Repeated Cross-Sectional Study. Nutrients 13: 24.

56. Hong SA, Peltzer K (2017) Dietary behaviour, psychological wellbeing and mental distress among adolescents in Korea. Child Adolesc Psychiatry Ment Health 11: 56.

57. Bazrafshan MR, Jokar M, Shokrpour N, Delam H (2020) The effect of lavender herbal tea on the anxiety and depression of the elderly: A randomized clinical trial. Complement Ther Med 50: 102393.

58. Perspective Global on 1012 nationally representative UK adults 2021.

59. Chung M, Zhao N, Wang D, White MS, Karlsen M, et al. (2020) Dose-Response Relation between Tea Consumption and Risk of Cardiovascular Disease and All-Cause Mortality: A Systematic Review and Meta-Analysis of Population-Based Studies. Adv Nutr 11: 790-814.

60. PHE, Public Health England (2018) The Eatwell Guide. Helping you eat a healthy, balanced diet 2018. 